\title{
BIOFILM FORMATION BY PERSISTENT AND NON-PERSISTENT LISTERIA MONOCYTOGENES STRAINS ON ABIOTIC SURFACES
}

\author{
R. Magalhães, V. Ferreira, G. Biscottini, T.R.S. Brandão, G. Almeida and P. Teixeira* \\ CBQF - Centro de Biotecnologia e Química Fina - Laboratório Associado, Escola Superior de Biotecnologia, \\ Universidade Católica Portuguesa, Rua Arquiteto Lobão Vital, Apartado 2511, 4202-401 Porto. Portugal
}

(Received: 6 May 2016; accepted: 13 July 2016)

Contaminated food with Listeria monocytogenes is the predominant route of transmission of listeriosis to humans, a severe illness with a high mortality rate. Food processing environments can be colonized by persistent strains, repeatedly isolated for months or years. This study aimed to investigate the biofilm formation capacity of six strains of L. monocytogenes that persisted for long periods in two cheese processing plants in comparison to seven strains isolated sporadically from the same plants. Persistent strains produced more biofilm than non-persistent strains on stainless steel and silicon rubber surfaces; no significant differences were observed on polyvinyl chloride (PVC). In a polystyrene microtiter plate assay with crystal violet staining, no evidence was found that persistent strains have higher ability to form biofilm than non-persistent strains, and no correlation was identified between biofilm formation in the microtiter plate and on the three other surfaces tested.

Keywords: Listeria monocytogenes, persistence, biofilm, stainless steel, silicon, PVC

Listeria monocytogenes is a Gram-positive bacterium responsible for causing listeriosis in humans. Although it is a rare disease, with a relatively low incidence $(0.52$ cases of invasive listeriosis per 100000 population in 2014), listeriosis is a severe disease in terms of hospitalization, with the highest case fatality rate $(15.0 \%)$ of all the zoonotic diseases under EU surveillance (EFSA\& ECDC, 2015). Contaminated food represents the major transmission route of this pathogen. Its common environmental distribution together with its unusual ability to adapt and survive under extreme conditions, as those inflicted by the environmental stresses of food processing plant, makes the development of effective strategies to control L. monocytogenes crucial. Cross-contamination by the equipment and general environment within the processing plant, after the foods have been processed, have been pointed out as one of the most important sources of food product contamination (LAPPI et al., 2004; AlmEIDA et al., 2013).

Several studies have demonstrated the colonization for long time periods (months or even years) of food processing plants by L. monocytogenes isolates presenting indistinguishable molecular subtypes (FERREIRA et al., 2011; AlmEIDA et al., 2013). These have been denominated persistent or dominant strains, while others that are recovered sporadically from the food environment are considered non-persistent or transient strains. This prolonged persistent contamination is not currently fully understood, and the hypothesis that persistent strains present distinct phenotypic traits, that allow their survival for long periods has been suggested. However, until now no strong evidence has been provided to validate this association (reviewed by FERREIRA and co-workers, 2014).

\footnotetext{
* To whom correspondence should be addressed.

Phone: +351 22558 0001; fax: +351 22509 0351; e-mail: pcteixeira@porto.ucp.pt
} 
Persistence of $L$. monocytogenes in the processing environment could be possible related to a higher ability to form biofilms where cells would be protected from the environmental stresses, e.g. L. monocytogenes cells in biofilm present increased resistance to antimicrobial agents and disinfectants than in the planktonic forms (NORWOOD \& GILMOUR, 2000; STOPFORTH et al., 2002).

The importance of research directed toward the elucidation of L. monocytogenes persistence is a crucial step for the development of effective and practical control strategies, targeting hygienic and sanitary issues, and decrease the likelihood of cross-contamination, such as the "seek-and-destroy" approach referred by MALLEY and co-workers (2015).

In the present study we analysed $13 \mathrm{~L}$. monocytogenes strains, selected from a larger set of isolates, previously characterised by different genotypic and phenotypic methods (MAGAlhÃES et al., 2016). These were randomly chosen to evaluate the ability of persistent and non-persistent L. monocytogenes strains to form biofilm on different abiotic surfaces, including: stainless steel, silicon rubber, and polyvinyl chloride (PVC). Biofilm formation by all strains using a microtiter plate assay was also investigated.

\section{Materials and methods}

\subsection{Listeria monocytogenes strains and inoculum preparation}

A set of persistent and non-persistent L. monocytogenes strains were selected from Listeria Research Centre from Escola Superior de Biotecnologia (LRCESB) culture collection based on previous study by ALMEIDA and co-workers (2013) that evaluated during a four-year period the contamination by L. monocytogenes in the environment, raw material, and final-products of different cheese-processing plants. Isolates collected in that study were characterised by DNA macrorestriction analysis by pulsed field gel electrophoresis (PFGE). The recurrent isolation of $L$. monocytogenes isolates with undistinguished molecular PFGE types on different dates and origins indicated evidence of in-house persistence in an artisanal producer of raw ewe's milk cheeses (APC) and in a small-scale industrial cheese producer (SSI). For the present study, six strains were randomly selected to represent PFGE types $\mathrm{Da}, \mathrm{Db}$, and Dc (recurrently isolated during 15, 9, and 8 months, respectively) from APC producer, and PFGE types $\mathrm{Ka}, \mathrm{Kb}$, and $\mathrm{E}$ (recurrently isolated over a period of four, three, and four years, respectively) from SSI producer (Table 1); these were denominated persistent strains. These strains were characterised in a previous study by MAGALHÃES and co-workers (2016), in which several isolates from each persistent PFGE type recovered from these two processing plants were evaluated concerning disinfectant resistance and kinetic behaviour at different temperatures. As no significant differences were observed among isolates belonging to the same persistent PFGE type, for this study, single strains representative of each PFGE type were selected. Additionally, seven L. monocytogenes strains that were isolated only once during the period of study (denominated non-persistent strains) in both processing plants were also included (Table 1). 


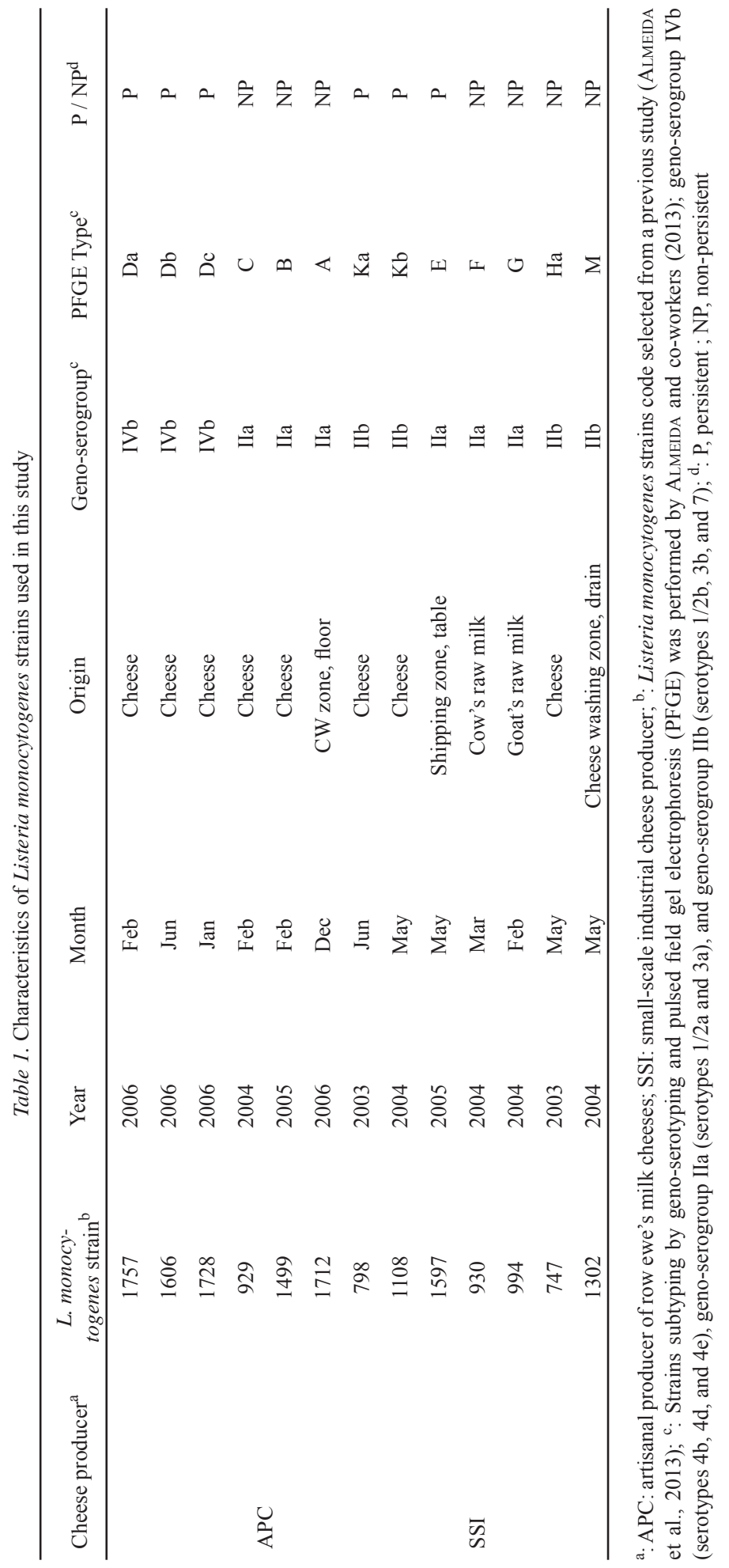


Stock cultures were kept in tryptic soya broth with yeast extract $0.6 \%$ (w/v) (TSBYE, Lab M, Heywood, Lanchashire, UK) supplemented with $30 \%$ (w/v) of glycerol at $-80{ }^{\circ} \mathrm{C}$. Before use, frozen stocks were streaked onto tryptic soya agar with yeast extract $0.6 \% \mathrm{w} / \mathrm{v}$ (TSAYE, Lab M) and incubated at $37^{\circ} \mathrm{C}$ overnight. A single colony was inoculated into 10 $\mathrm{ml}$ of TSBYE and incubated overnight at $37^{\circ} \mathrm{C}$. The cultures were then sub-cultured in $10 \mathrm{ml}$ of TSBYE $(1 \% \mathrm{v} / \mathrm{v})$ and incubated at the same conditions. The optical density (OD) of each cell suspension was further measured at $600 \mathrm{~nm}$, and inoculum was prepared by dilution in TSB to obtain approximately $10^{6}$ cells per $\mathrm{ml}$.

\subsection{Preparation of stainless steel, silicon rubber and PVC coupons}

Stainless steel (type 304) and silicon rubber coupons $(2.4 \mathrm{~cm} \times 7.3 \mathrm{~cm})$ were washed with distilled water and immersed for $3 \mathrm{~min}$ in acetone, followed by a second wash with distilled water and immersed for $3 \mathrm{~min}$ in ethanol $70 \%(\mathrm{v} / \mathrm{v})$. Coupons were then rinsed with distilled water, air-dried, and sterilized at $121{ }^{\circ} \mathrm{C}$ for $15 \mathrm{~min}$. PVC coupons $(2.4 \mathrm{~cm} \times 7.3 \mathrm{~cm})$ were washed with distilled water, air-dried, and ethylene oxide-sterilized. The sterile coupons were then immersed vertically in $50 \mathrm{ml}$ sterile Falcon tubes (Sarstedt, Nümbrecht, Germany) previously filled with $45 \mathrm{ml}$ of sterile TSB (LabM). All coupons were fitted into Falcon tubes so that both sides were available for bacterial adherence.

\subsection{Evaluation of biofilm forming ability in stainless steel, silicon rubber, and PVC coupons by cell enumeration}

Three sets of three tubes were set up with each surface material to be tested (i.e. stainless steel, silicon rubber, or PVC), each containing a single coupon, and inoculated with the different strains to achieve a test suspension with approximately $10^{6}$ cells per ml. Tubes were submitted to static incubation for 5 days at $22^{\circ} \mathrm{C}$; the growth medium was selected based on previous studies that showed acceptable results for biofilm formation by $L$. monocytogenes using TSB (HARveY et al., 2007; LATORRE et al., 2011), and temperature of $22{ }^{\circ} \mathrm{C}$ was chosen to represent room temperature. The coupons were then rinsed twice with sterile distilled water and transferred aseptically into a new tube containing $10 \mathrm{ml}$ of sterile phosphate buffer saline (PBS). Biofilms were removed by swabbing on both sides followed by vortexing for 1 min. Serial decimal dilutions were prepared in PBS and inoculated in sterile Petri dish with TSAYE by the drop technique (according to MiLES \& MisRA, 1938) in duplicate. Plates were incubated overnight at $37{ }^{\circ} \mathrm{C}$, and colony enumeration was performed. Two independent experiments were conducted for each surface material (each one with a set of three tubes). Pseudomonas aeruginosa ATCC 10145 was used in each assay as a positive control (strong biofilm former; MoHSEN et al., 2015). A negative control (tubes with coupons and media without bacteria) was also included. Biofilm formation was determined by calculation of $\log _{10}$ of colony-forming units (CFU) per $\mathrm{cm}^{2}$.

\subsection{Quantification of biofilm by microtiter plate assay}

Evaluation of the ability of each strain to form biofilm by microtiter plate assay was performed using a 96 wells sterile polystyrene flat bottom microplate (Orange Scientific, Braine-l'Alleud, Belgium) according to CHRISTENSEN and co-workers (1985), with minor modifications. Briefly, for each strain six wells were filled with $200 \mu \mathrm{l}$ of inoculum with approximately $10^{6}$ cells per $\mathrm{ml}$. Six wells with sterile TSB were included as controls. Following static incubation at $22^{\circ} \mathrm{C}$ for 5 days, biofilm formation was assessed by crystal violet staining (Merck, Darmstadt, 
Germany). Growth media was removed and wells were gently washed three times with $250 \mu \mathrm{l}$ of sterile distilled water to remove unattached cells. Attached cells were further fixed using $250 \mu \mathrm{l}$ of methanol solution (Merck) per well for $15 \mathrm{~min}$ and left to air-dry. Crystal violet was added [50 $\mu$ l of a $0.1 \%(\mathrm{v} / \mathrm{v})$ solution] to each well and allowed to stain for $45 \mathrm{~min}$ at room temperature. Excess stain was removed by gently washing the microplate under running tap water. Microplates were air-dried and $250 \mu 1$ of a $33 \%$ (v/v) acetic acid solution (Merck) was added to each well and incubated for $30 \mathrm{~min}$ at $4{ }^{\circ} \mathrm{C}$. Subsequently, $100 \mu \mathrm{l}$ from each well were transferred into a new microplate and optical density (OD) was read at $595 \mathrm{~nm}$ using a microplate reader (Model 680, Bio-Rad, Hercules, CA, USA). Two independent experiments were performed; for each strain OD values were averaged.

\subsection{Statistical analysis}

Listeria monocytogenes strains were categorized into two groups: persistent strains and nonpersistent strains. Aiming at concluding about significant difference in relation to strains in biofilm formation ability $\left(\log _{10} \mathrm{CFU} \mathrm{cm} \mathrm{cm}^{-2}\right.$ and $\left.\mathrm{OD}_{595}\right)$, a Student $t$-test was performed for comparison of means assuming independent samples. Normality and homoscedasticity was assessed for all groups using Kolmogorov-Smirnov and Levene's tests, respectively. The significance level assumed was $5 \%$ in all situations. Analyses were performed using IBM SPSS ${ }^{\circledR}$ Statistics ${ }^{\circledR} 20$ for Windows ${ }^{\circledR}$ (SPSS Inc., Chicago, USA).

\section{Results and discussion}

Values of $\log _{10} \mathrm{CFU} \mathrm{cm} \mathrm{cm}^{-2}$ obtained for persistent $(\mathrm{n}=6)$ and non-persistent $(\mathrm{n}=7)$ strains for each material and microtiter plate assay for the 13 strains are presented in Table 2 and Figure 1. P. aeruginosa ATCC 10145 presented the highest values on all tested surfaces. Significant differences in biofilm formation were found between persistent and non-persistent strains on two of the surfaces tested; overall, persistent strains were better biofilm formers on stainless steel $(\mathrm{P}=0.018)$ and on silicon rubber $(\mathrm{P}=0.025)$. In $\mathrm{PVC}$ coupons no relation between persistence and higher ability to form biofilm was found $(\mathrm{P}=0.141)$. Overall, the quantity of biofilm produced by each strain was similar on stainless steel and silicon rubber coupons $(\mathrm{P}=0.914)$ but different on $\mathrm{PVC}(\mathrm{P}<0.001)$; the majority of the strains presented higher $\log _{10}$

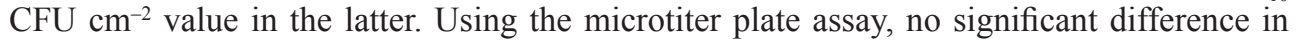
biofilm formation was found between persistent and non-persistent strains $(\mathrm{P}=0.059)$, and no significant relationship was found with results obtained using the three other surfaces.

Several authors have compared biofilm formation between persistent and non-persistent strains of L. monocytogenes isolated from food-contact environments, generating diverse and conflicting results. To our knowledge, nine studies on the subject have been reported so far, all of them using a single methodology: in one study a polystyrene microtiter assay was used (HARvey et al., 2007), in two studies stainless steel coupons were used (Norwood \& Gilmour, 1999; LundÉn et al., 2000), while in the remaining five a PVC microtiter assay was selected (Djordjevic et al., 2002; Borucki et al., 2003; Jensen et al., 2007; Cruz \& Fletcher, 2011; LATORRE et al., 2011; WANG et al., 2015). The culture media, growth conditions, time to allow biofilm formation, and temperatures were diverse. Similarly to our results, Norwood and GiLmour (1999) found a statistically significant relationship between persistence of $L$. monocytogenes and ability to form biofilm on stainless steel coupons, as well as BORUCKI and co-workers (2003) and LATORRE and co-workers (2011) using PVC microtiter assays. HARVEY 
and co-workers (2007) using, like in our study, a polystyrene microtiter assay, found no significant differences in biofilm formation among persistent and non-persistent strains; the same conclusion was drawn by the other studies (LUNDÉn et al., 2000; DJORDJEVIC et al., 2002; Jensen et al., 2007; Cruz \& Fletcher, 2011; WANG et al., 2015). It is very important to keep in mind that conclusions drawn from the different studies are always deeply correlated not only with the experimental conditions and strains, as detailed above, but also with the concept of "persistence" itself, as the criteria used to identify persistent and non-persistent strains is not consistent among studies.

Table 2. Biofilm formation for persistent (P) and non-persistent (NP) L. monocytogenes isolates and P. aeruginosa on abiotic surfaces tested and in the microtiter plate assay

\begin{tabular}{lcccc}
\hline P/NP & Strain & \multicolumn{3}{c}{$\log _{10} \mathrm{CFU} \mathrm{cm}(\mathrm{Mean} \pm \mathrm{SD})$} \\
\cline { 3 - 5 } & & Stainless steel & Silicone & $\mathrm{PVC}$ \\
\hline $\mathrm{P}$ & $3.52 \pm 0.21$ & $2.90 \pm 0.43$ & $3.82 \pm 0.51$ \\
& 798 & $3.27 \pm 0.40$ & $3.43 \pm 0.24$ & $3.94 \pm 0.41$ \\
& 1108 & $3.38 \pm 0.21$ & $3.88 \pm 0.56$ & $3.69 \pm 0.32$ \\
1597 & $3.58 \pm 0.17$ & $3.48 \pm 0.11$ & $3.60 \pm 0.56$ \\
& 1606 & $4.22 \pm 0.39$ & $3.65 \pm 0.37$ & $3.92 \pm 0.34$ \\
& 1728 & $3.61 \pm 0.15$ & $3.75 \pm 0.20$ & $3.53 \pm 0.32$ \\
\hline $\mathrm{NP}$ & $3.68 \pm 0.23$ & $3.52 \pm 0.14$ & $3.85 \pm 0.17$ \\
& 7457 & $3.64 \pm 0.16$ & $3.49 \pm 0.32$ & $3.95 \pm 0.49$ \\
& 929 & $3.16 \pm 0.45$ & $3.52 \pm 0.32$ & $3.97 \pm 0.63$ \\
& 930 & $3.03 \pm 0.48$ & $3.06 \pm 0.34$ & $4.41 \pm 0.41$ \\
& 994 & $2.80 \pm 0.08$ & $3.16 \pm 0.45$ & $3.11 \pm 0.24$ \\
& 1302 & $3.40 \pm 0.46$ & $3.03 \pm 0.40$ & $4.38 \pm 0.61$ \\
& 1499 & $3.48 \pm 0.51$ & $3.32 \pm 0.34$ & $3,77 \pm 0.15$ \\
& 1712 & $6.31 \pm 0.12$ & $6.58 \pm 0.04$ & $6.47 \pm 0.13$ \\
\hline
\end{tabular}

P: Persistent; NP: non-persistent

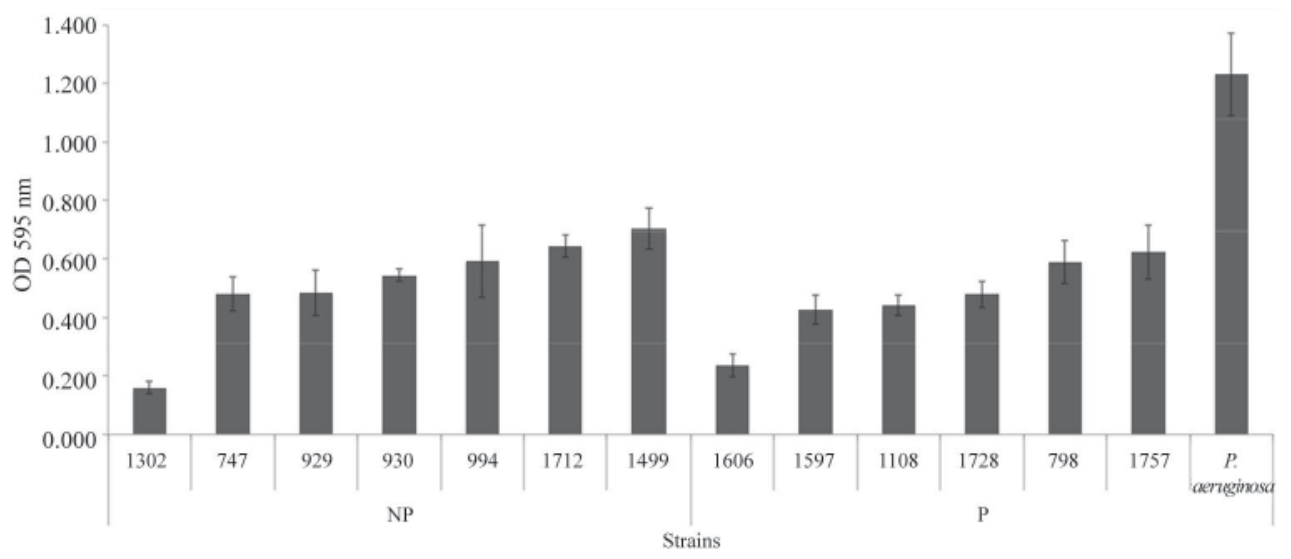

Fig. 1. Values of absorbance at $595 \mathrm{~nm}$ obtained for persistent (P) and non-persistent (NP) L. monocytogenes isolates and $P$. aeruginosa for biofilm formation incubated at $22{ }^{\circ} \mathrm{C}$ for 5 days 
The microtiter plate assay has been widely used to screen differences in biofilm formation among different species, including L. monocytogenes isolates. Although it offers significant advantages such as small sample volumes, simultaneous analysis of multiple strains, and rapid turnaround, when compared to other cost- and labour-intensive techniques, drawing inferences from results obtained to estimate the behaviour of L. monocytogenes in the surfaces encountered in the processing environment should be taken carefully.

Despite the statistically significant differences obtained in this study, the mean values obtained for persistent and non-persistent strains are quite similar, and a high variability among strains was observed; therefore, the biological significance of these results is unclear. The development of a realistic methodology reflecting the true ability of $L$. monocytogenes to form biofilm in food processing environments is virtually impossible, due to the complexity and diversity of factors involved; however, it would be valuable to establish a standard reproducible method for biofilm quantification among different strains to avoid experimental variables among different studies, other than strain inter-specificity.

\section{Conclusions}

In this study, biofilm formation by L. monocytogenes was dependent on surface materials. Overall, strains tested presented higher ability to form biofilm on PVC, followed by stainless steel and silicon rubber; the number of adhered cells was not significantly different between these two last materials. No correlation was identified between biofilm formation using a microtiter plate assay with crystal violet staining and on the three other surfaces tested. Persistent strains were better biofilm formers on stainless steel and on silicon rubber, while on PVC and in the microtiter assay no relation between persistence and higher ability to form biofilms was found.

This work was supported by funding from the National Funds from FCT - Fundação para a Ciência e a Tecnologia through project UID/Multi/50016/2013 and "KLEAR - Knowledge and tools for safety, quality and competitiveness in the food chain." $\mathrm{n}^{\mathrm{o}}$ NORTE-07-0124-FEDER-000052, co-funded by Fundo Europeu de Desenvolvimento Regional (FEDER), under Programa ON2, Eixo Prioritário 01-01 - Competitividade, Inovação e Conhecimento, Tipologia 01-01-01-24 - Apoio a Entidades do Sistema Científico e Tecnológico. Financial support for authors Rui Magalhães and Vânia Ferreira was provided by FCT through doctoral fellowship SFRH/BD/71704/2010 and postdoctoral fellowship SFRH/BPD/72617/2010, respectively.

\section{References}

Almeida, G., Magalhães, R., Carneiro, L., Santos, I., Silva, J., Ferreira, V., Hogg, T. \& Teixeira, P. (2013): Foci of contamination of Listeria monocytogenes in different cheese processing plant. Int. J. Food Microbiol., 167, 303-309.

Borucki, M.K., Peppin, J.D., White, D., Loge, F. \& Call, D.R. (2003): Variation in biofilm formation among strains of Listeria monocytogenes. Appl. Environ. Microb., 69, 7336-7342.

Christensen, G.D., Simpson, W.A., Younger, J.J., Baddour, L.M., Barrett, F.F., Melton, D.M. \& Beachey, E.H. (1985): Adherence of coagulase-negative Staphylococci to plastic tissue culture plates: A quantitative model for the adherence of Staphylococci to medical devices. J. Clin. Microbiol., 22, 996-1006.

Cruz, C.D. \& Fletcher, G.C. (2011): Prevalence and biofilm-forming ability of Listeria monocytogenes in New Zealand mussel (Perna canaliculus) processing plants. Food Microbiol., 28, 1387-1393. 
Djordjevic, D., Wiedmann, M. \& McLandsborough, L.A. (2002): Microtiter plate assay for assessment of Listeria monocytogenes biofilm formation. Appl. Environ. Microb., 68, 2950-2958.

EFSA (European Food Safety Authority) \& ECDC (European Centre for Disease Prevention and Control) (2015): The European Union summary report on trends and sources of zoonoses, zoonotic agents and foodborne outbreaks in 2014. EFSA J., 13, 4329, 191 pages.

Ferreira, V., Barbosa, J., Stasiewicz, M., Vongkamjan, K., Moreno, S.A., Hogg, T., Gibbs, P., Teixeira, P. \& Wiedmann, M. (2011): Diverse geno- and phenotypes of persistent Listeria monocytogenes isolates from fermented meat sausage production facilities in Portugal. Appl. Environ. Microb., 77, 2701-2715.

Ferreira, V., Wiedmann, M., Teixeira, P. \& Stasiewicz, M.J. (2014): Listeria monocytogenes persistence in foodassociated environments: Epidemiology, strain characteristics, and implications for public health. J. Food Protect., 77, 150-170.

Harvey, J., Keenan, K. \& Gilmour, A. (2007): Assessing biofilm formation by Listeria monocytogenes strains. Food Microbiol., 24, 380-392.

Jensen, A., Larsen, M.H., Ingmer, H., Vogel, B.F. \& Gram, L. (2007): Sodium chloride enhances adherence and aggregation and strain variation influences invasiveness of Listeria monocytogenes strains. J. Food Protect., 70, 592-599.

Latorre, A.A., Van Kessel, J.A., Karns, J.S., Zurakowski, M.J., Pradhan, A.K., Boor, K.J., Adolph, E., SukhnANAND, S. \& SchukKen, Y.H. (2011): Increased in vitro adherence and on-farm persistence of predominant and persistent Listeria monocytogenes strains in the milking system. Appl. Environ. Microb., 77, 3676-3684.

Lappi, V.R., Thimothe, J., Nightingale, K.K., Gall, K., Scott, V.N. \& Wiedmann, M. (2004): Longitudinal studies on Listeria in smoked fish plants: impact of intervention strategies on contamination patterns. J. Food Protect., 67, 2500-2514.

Lundén, J.M., Miettinen, M.K., Autio, T.J. \& Korkeala, H.J. (2000): Persistent Listeria monocytogenes strains show enhanced adherence to food contact surface after short contact times. J. Food Protect., 63, 1204-1207.

Malley, T.J., Butts, J. \& Wiedmann, M. (2015): Seek and destroy process: Listeria monocytogenes process controls in the ready-to-eat meat and poultry industry. J. Food Protect., 78, 436-445.

Magalhães, R., Ferreira, V., Brandão, T.R.S., Casquete Palencia, R., Almeida, G. \& Teixeira, P. (2016): Persistent and non-persistent strains of Listeria monocytogenes: a focus on growth kinetics under different temperature, salt, and pH conditions and their sensitivity to sanitizers. Food Microbiol., 57, 103-108.

Miles, A.A. \& Misra, S.S. (1938): The estimation of the bactericidal power of blood. J. Hygiene, 38, 732-749.

Mohsen, A., Gomaa, A., Mohamed, F., Ragab, R., Eidm, Ahmed A., Khalaf, A., Kamal, M., Mokhtar, S., Mohamed, H., Salah, I., Abbas, R. \& Ali, S. (2015): Antibacterial, anti-biofilm activity of some non-steroidal antiinflammatory drugs and $\mathrm{N}$-acetyl cysteine against some biofilm producing uropathogens. Am. J. Epidemiol. Infect. Dis. (AJED), 3, 1-9.

Norwood, D. \& Gilmour, A. (1999): Adherence of Listeria monocytogenes strains to stainless steel coupons. J. Appl. Microbiol., 86, 576-582.

Norwood, D. \& Gilmour, A. (2000): The growth and resistance to sodium hypochlorite of Listeria monocytogenes in a steady-state multispecies biofilm. J. Appl. Microbiol., 88, 512-520.

Stopforth, J.D., Samelis, J., Sofos, J.N., Kendall, P.A. \& Smith, G.C. (2002): Biofilm formation by acid-adapted and nonadapted Listeria monocytogenes in fresh beef decontamination washings and its subsequent inactivation with sanitizers. J. Food Protect., 65, 1717-1727.

Wang, J., RaY, A.J., Hammons, S.R. \& Oliver, H.F. (2015): Persistent and transient Listeria monocytogenes strains from retail deli environments vary in their ability to adhere and form biofilms and rarely have inla premature stop codons. Foodborne Pathog. Dis., 12, 151-158. 